\title{
Collaborative Beamforming Techniques for Data Transmission in Wireless Sensor Networks
}

\author{
Lazar Berbakov and Marko Beko
}

\begin{abstract}
Collaborative beamforming is a technique for improving energy efficiency of uplink communications in wireless sensor networks. It is done by synchronizing carrier phases of individual sensor nodes precisely enough, so that they jointly form a beampattern with a stable mainlobe. In this paper, we analyze the impact of additive noise in the received signal on the behavior of an iterative phase synchronization scheme. Besides, we analyze the bit error rate performance of such collaborative transmission. In particular, we consider a decentralized antenna array where sensors are allowed to perform distributed carrier synchronization and collaborative data transmission simultaneously. We evaluate the system performance by using numerical simulations and provide a semi-analytical solution for the algorithm convergence characteristics and the overall bit error rate as a function of the algorithm's iteration index.
\end{abstract}

Keywords - Collaborative Beamforming, Wireless Sensor Networks.

\section{INTRODUCTION}

$\mathrm{R}$ ECENT improvements in microelectronics, wireless networking and embedded microprocessors have enabled a production of capable and cheap sensor nodes suitable for a range of commercial and military applications such as: home automation, healthcare applications, space exploration, battlefield surveillance, etc. It is expected that in the future Internet of Things, the objects in our environment will be able to communicate among themselves and make autonomous decisions without human supervision. With such a large number of battery operated devices, and possibly a distant base

Paper received April 8, 2015; revised August 5, 2015; accepted August 19, 2015. Date of publication November 15, 2015. The associate editor coordinating the review of this manuscript and approving it for publication was Prof. Aleksandar Nešković.

This paper is a revised and expanded version of the paper presented at the 22th Telecommunications Forum TELFOR 2014

The work of Lazar Berbakov was supported by grant TR32043 of the Ministry of Education, Science and Technological Development of the Republic of Serbia and Bilateral Cooperation Project Portugal-Serbia No. 451-03-02338/2012-14/11. The work of Marko Beko was partially supported by Fundação para a Ciência e a Tecnologia under Projects PEst- OE/EEI/UI0066/2014, PTDC/EEI-TEL/2990/2012 ADIN, EXPL/EEITEL/0969/2013 MANY2COMWIN and EXPL/EEITEL/1582/2013 GLANC, and Ciência 2008 Post-Doctoral Research grant.

Lazar Berbakov is with the Mihailo Pupin Institute, University of Belgrade, Volgina 15, 11050 Belgrade, Serbia; (phone +381 11677 5460; email: lazar.berbakov@pupin.rs).

Marko Beko is with Universidade Lusofona de Humanidades e Technologias, Lisbon, Portugal and UNINOVA - Campus FCT/UNL, Caparica, Portugal; (email: beko.marko@gmail.com). station (BS), energy efficiency becomes one of the most important topics of investigation in this area. Nevertheless, high density deployment of nodes in wireless sensor networks (WSNs) allows us to use collaborative beamforming $(\mathrm{CBF})$ techniques and effectively deal with the aforementioned energy constraint. Collaborative beamforming is particularly useful in applications such as environmental parameter monitoring, battlefield surveillance, where BS is usually located far away and each individual sensor node cannot reach it on its own due to power and battery constraints.

One of the prerequisites for collaborative beamforming is to have sensor nodes' oscillators synchronized in frequency and phase. This assumption, naturally assumed in centralized antenna arrays, turns out to be much trickier to achieve in a distributed system such as a WSN.

A carrier phase synchronization technique, suitable for simple hardware usually found in cheap sensor nodes, is described in [1]. In that paper, the authors propose a low complexity carrier phase synchronization algorithm requiring only one bit of feedback per iteration. In the proposed scheme, all the sensors make random phase perturbations of their carriers in each iteration. The sum received signal strength (RSS) is estimated at the destination and compared to the RSS from the previous iteration. Finally, the outcome of this comparison is fedback to all the sensors, telling them to either retain or discard the last phase perturbation. This procedure is repeated until the RSS reaches some predefined level. In [1], the authors assume that the noise can be neglected, which leads to the perfect RSS estimation at the BS. With this assumption, the authors were able to prove that the proposed algorithm converges almost surely to full phase synchronization. The algorithm in [1] is further generalized in [2], to allow simultaneous frequency and phase synchronization. Furthermore, it is improved in [3] by optimizing perturbation size in each iteration and in [4] where the authors also utilize negative feedback instead of simply discarding it.

A completely different approach to distributed beamforming is proposed in [5], where the authors use the fact that collaborative transmission with sensors that are not perfectly synchronized can still provide a significant beamforming gain. This fact allows them to develop an algorithm that is based on the selection of a subset of sensor nodes whose signals are combined in a quasicoherent manner at the destination. Work in [5] was further extended in [6], where the authors propose an algorithm that uses more feedback to tell selected nodes 
whether and how they should pre-compensate phases. Theoretical analysis provided therein shows that the proposed scheme is more effective when the number of relay nodes is large.

To the best of authors' knowledge, all the papers in the literature assume that the distributed phase synchronization is performed separately from the data transmission. Depending on the amount of energy consumed for carrier synchronization overhead, collaborative transmission may result in much lower energy efficiency.

In this paper, we provide the generalization of the convergence analysis from [1], for the case where noise at the BS cannot be neglected. This assumption, as will be shown here, prevents the algorithm from achieving full phase synchronization, and instead the RSS is shown to saturate to the value that depends on the noise power. In addition, here we propose a CBF scheme where carrier synchronization and data transmission are performed simultaneously. The proposed algorithm is a variation of the one presented in [1], but now, we allow sensors to transmit data with a modulation scheme that does not affect the distributed phase synchronization algorithm. The performance of the proposed algorithm is assessed in terms of RSS and the bit error rate (BER) as a function of algorithm's iteration.

The rest of the paper is organized as follows: Section II provides the overall signal and communication model. In Section III, we analyze the algorithm's behavior with noisy RSS measurements at the BS. Next, Section IV provides analysis of the algorithm with simultaneous carrier synchronization and data transmission. Finally, in Section V, we give some analytical and numerical results illustrating the impact of channel noise on the behavior of the algorithm and the performance of the proposed communication scheme.

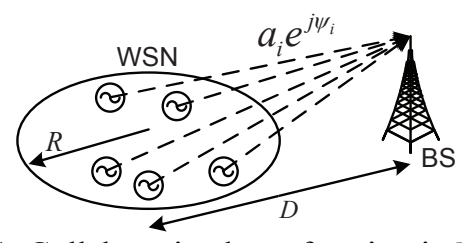

Fig. 1. Collaborative beamforming in WSN.

\section{SIGNAL AND COMMUNICATION MODEL}

Consider a WSN that consists of $N$ sensor nodes randomly deployed over a disk of radius $R$ according to a uniform distribution, as shown in Fig. 1. The goal is to collaboratively send a common message ${ }^{1} m(t)$ with $\mathbb{E}\left[|m(t)|^{2}\right]=1$, to a BS located on the XY plane at a distance $D>>R$ (i.e. far field conditions apply).

In order to save energy, the sensor nodes spend most of the time in the sleep mode. When new data needs to be collected, the BS transmits an RF signal to activate the carrier detectors at the sensor nodes and wakes them up (for more details, see [7]). It is assumed that the oscillators at the sensor nodes have a low frequency drift, so that after the sleep time, they remain frequency locked to the

\footnotetext{
${ }^{1}$ A source node can simply broadcast the common message to all the sensors in a given cluster.
}

reference carrier frequency $f_{c}$. The initial phase offset of each oscillator $\gamma_{i}$ is unknown, and it can be assumed as i.i.d. over sensors and uniformly distributed (i.e. $\gamma_{i} \sim \mathcal{V}(-\pi$, $\pi)$ ). The signal transmitted by the $i$-th sensor node is given by:

$$
s_{i}(t)=g_{i}^{*} m(t) e^{j\left(2 \pi f_{c} t+\gamma_{i}\right)},
$$

with $g_{i}=b_{i} e^{j \theta_{i}}$ denoting the corresponding transmit weight (to be designed). The complex sensor-to-BS channel is denoted by $h_{i}=a_{i} e^{j \psi_{i}}$, where $a_{i}$ and $\psi_{i}$ stand for the channel magnitude and phase shift associated to the Euclidian distance between the corresponding sensor and the BS, respectively. In this paper, we assume that neither BS nor sensor nodes have the knowledge of $h_{i}$.

Unlike previous works on collaborative beamforming [1], in this paper we assume that the data transmission is performed simultaneously with the carrier phase synchronization. This is possible since the algorithm for carrier synchronization proposed in [1] requires only RSS measurements at the BS, allowing us to simultaneously transmit data by using a PSK modulation scheme (e.g. BPSK or QPSK). Consequently, the convergence rate of the carrier synchronization algorithm remains unchanged.

The PSK modulated message signal is given by $m(t)=e^{j v[n]}$ for $t=\left[(n-1) T_{I}, n T_{I}\right), \quad$ where $v[n] \in\left\{\varphi_{1}\right.$, $\left.\varphi_{1}+\pi\right\}$ for BPSK and $v[n] \in\left\{\varphi_{1}, \varphi_{1}+\pi / 2, \varphi_{1}+\pi\right.$, $\left.\varphi_{1}+3 \pi / 2\right\}$ for QPSK, with $T_{I}$ denoting the timeslot duration and $\varphi_{1}$ standing for the initial phase offset of the modulation signal. When all the sensors send common data collaboratively, the signal received at the BS during the $n$-th iteration can be written as:

$$
r(t)=e^{j 2 \pi f_{c} t} \sum_{i=1}^{N} a_{i} b_{i} e^{j\left(\gamma_{i}+\psi_{i}-\theta_{i}+v[n]\right)}+w(t)
$$

where $w(t) \sim C \mathcal{N}\left(0, \sigma_{w}^{2}\right)$ denotes additive white Gaussian noise (AWGN). Due to hardware limitations, the transmit power at each sensor node is assumed to be constant and, hence, the transmit weights read $g_{i}=e^{j \theta_{i}}$. In addition, since the distance between the BS and the sensors is much larger than inter-sensor distances (in addition to line-ofsight conditions), we also assume that $a_{i}=1$ for all sensors. After down-conversion and sampling, the RSS at the BS in time instant $n$ reads:

$$
\operatorname{RSS}[n]=R[n]=\mid \sum_{i=1}^{N} e^{j \Phi_{i}[n]}+w[n],
$$

where $\Phi_{i}[n]=\gamma_{i}+\psi_{i}-\theta_{i}[n]+v[n]$ denotes the overall phase rotation of the signal received at the BS from the $i$ th sensor node whereas $\theta_{i}[n]$ accounts for a time varying beamforming weight adjusted by adding a random phase perturbation $\delta_{i}[n]$ in each algorithm iteration in order to maximize $^{2}$ the RSS. Note that the message signal $m(t)$ impacts only the received signal phase through the factor $v[n]$, whereas the signal magnitude remains to be the same

\footnotetext{
${ }^{2}$ The optimal numerical solution for the problem where the RSS at the BS is maximized requires knowledge of all the sensor-to-BS channels and it can be found in [8].
} 
as in the case without modulation.

We define the beamforming gain $Y[n]$ as:

$$
Y[n]=\left|\sum_{i=1}^{N} e^{j \Phi_{i}[n]}\right| \in \mathbb{R}^{+} \cup\{0\},
$$

and, accordingly, we can write

$$
R[n]=\left|Y[n]+w[n] e^{-j \alpha[n] \mid}\right| \stackrel{\Delta}{=} \mid Y[n]+w_{\alpha}[n],
$$

with $\alpha[n]=\arg \left\{\sum_{i=1}^{N} e^{j \Phi_{i}[n]}\right\}$ and where $w_{\alpha} \sim \operatorname{CN}\left(0, \sigma_{w}^{2}\right)$. Clearly, the random variable $R$ is Ricean distributed. However, for $Y>\sigma_{w}$ its probability density function (p. d. f.) can be approximated by a Gaussian distribution [9], namely,

$$
R \sim \mathcal{N}\left(\sqrt{Y^{2}[n]+\frac{\sigma_{w}^{2}}{2}}, \frac{\sigma_{w}^{2}}{2}\right)
$$

\section{Distributed BEAMFORMING SCHEME WITH NOISY RSS MEASUREMENTS}

Clearly, the beamforming gain of (4) is maximized when the individual signals are coherently combined, namely, $\gamma_{i}+\psi_{i}-\theta_{i}[n]+v[n]=C ; \quad \forall i$ (where $C$ is a constant) which yields $Y[n]=Y_{\max }=\left|\sum_{i=1}^{N} 1\right|=N$. To that aim, sensors must pre-compensate the unknown oscillator and channel phase offsets by properly adjusting the $\theta_{i}$ term during a training period. In the collaborative beamforming scheme of [1] this is achieved in an iterative manner.

Let us for the moment assume that no modulation signal is present $(v[n]=0)$, so that we can focus exclusively on the phase synchronization algorithm. Initially, the phases of the received signals at the base station, $\Phi_{i}[0]=\gamma_{i}+\psi_{i}-\theta_{i}[0]=\gamma_{i}+\psi_{i}$, are uniformly distributed in $[-\pi, \pi]^{3}$. At all times, each transmitter keeps track of the best value of $\theta_{\text {best }, i}[n]$. At each iteration, $\theta_{\text {best }, i}[n]$ is adjusted with a random perturbation $\delta_{i}[n]$ drawn from a suitable probability distribution $f_{\delta}\left(\delta_{i}\right)$. Next, all the sensor nodes transmit their signals with the incremental phase rotations, namely, $\theta_{i}[n+1]=\theta_{\text {best }, i}[n]+\delta_{i}[n]$ and the BS measures the RSS again. By comparing $R[n+1]$ with $R_{\text {best }}[n]=\max _{m \leq n} R[m]$ (i.e. the largest RSS until time instant $n$ ), the BS determines whether the set of perturbations $\Delta[n]=\left[\delta_{1}[n], \ldots, \delta_{N}[n]\right]$ should be kept (if RSS increases) or discarded (otherwise). The BS notifies the sensor nodes about this decision by sending one bit of feedback over an error-free common signalling channel. More formally, the sensor nodes update their phases according to the following recursion:

$$
\theta_{\text {best }, i}[n+1]= \begin{cases}\theta_{\text {best }, i}[n]+\delta_{i}[n] & \text { if } R[n+1] \geq R_{\text {best }}[n], \\ \theta_{\text {best }, i}[n] & \text { otherwise }\end{cases}
$$

As a result, the beamforming gain $Y[n]$ is updated as follows:

$$
Y_{\text {best }}[n+1]=\left\{\begin{array}{ll}
Y[n+1] & \text { if } R[n+1] \geq R_{\text {best }}[n], \\
Y_{\text {best }}[n] & \text { otherwise }
\end{array},\right.
$$

\footnotetext{
${ }^{3}$ This follows from the fact that, on the one hand, the oscillators operate independently and, on the other, $R<<D$.
}

and a similar expression applies to $R_{\text {best }}[n]$. It is worth noting that, unlike in [1], the decision is made on the basis of the noisy random variable $R[n]$. This means that, even if RSS increase, i.e. $R[n+1] \geq R_{\text {best }}[n]$, the system could experience a decrease in terms of beamforming gain (i.e. $\left.Y_{\text {best }}[n+1]<Y_{\text {best }}[n]\right)$. This has a number of implications that will be discussed in subsequent sections.

\section{A. Evolution of the expected beamforming gain}

From [1], we know that the following equality holds for the expected value of the normalized beamforming gain:

$$
\frac{1}{N} \mathbb{E}\left[Y_{\text {best }}[n+1]\right]=\frac{1}{N} \mathbb{E}\left[Y_{\text {best }}[n]\right]+\mathbb{E}\left[h_{n}(y)\right],
$$

with the random variable $y$ being defined as $y=Y_{\text {best }}[n] / N$. For the sake of clarity, the expectations in the above equation are taken with respect to the random perturbations and the measured received signal strength in time instants $n$ and $n+1$, where relevant; and the function $h_{n}(y)$ denotes the conditional expectation of the increment in the normalized beamforming gain, given that the normalized beamforming gain from previous iteration equals $1 / N \cdot Y_{\text {best }}[n]=y$, namely,

$$
\begin{aligned}
& h_{n}(y) \stackrel{\Delta}{=} \frac{1}{N} \mathbb{E}\left[\left(Y[n+1]-Y_{\text {best }}[n]\right) \times\right. \\
& \left.\times \mathcal{H}\left(R[n+1]-R_{\text {best }}[n]\right) \mid \frac{1}{N} Y_{\text {best }}[n]=y\right],
\end{aligned}
$$

with $\mathcal{H}(x)$ denoting the Heaviside step function:

$$
\mathcal{H}(x)=\left\{\begin{array}{ll}
1 & x>0 \\
0 & \text { otherwise }
\end{array},\right.
$$

which models the acceptance rule of phase perturbations given by (7) and (8). From (10) and based on the observation that $Y_{\text {best }}[n]$ is highly concentrated around its expected value when $N$ is large, we have that

$$
\frac{1}{N} \mathbb{E}\left[Y_{\text {best }}[n+1]\right] \approx \frac{1}{N} \mathbb{E}\left[Y_{\text {best }}[n]\right]+h_{n}\left(\frac{1}{N} \mathbb{E}\left[Y_{\text {best }}[n]\right]\right) \text {. }
$$

The last equation suggests that the evolution of the expected (and normalized) beamforming gain can be modeled through function $h_{n}(y)$. In the sequel, we attempt to derive an expression for such a function that, unlike in previous works [1], explicitly takes into consideration the impact of noise.

The authors in [1] showed that, given $Y_{\text {best }}[n] / N=y$, the following holds for normalized beamforming gain in the $(n+1)$-th iteration

$$
\frac{1}{N} Y_{b e s t}[n+1] \underset{p}{\longrightarrow} X_{n} y+x
$$

where $X_{n}=1-\operatorname{Var}\left[\delta_{\mathrm{i}}[n]\right] / 2$ is constant, and $x$ denotes a zeromean Gaussian r.v. with variance:

$$
\sigma_{\mathbb{R}}^{2}[n]=\frac{1-X_{n}^{2}-\rho_{n} \kappa_{n}(y)}{2 N},
$$

with $\kappa_{n}(y)$ standing for a function of the normalized beamforming gain y only which can be approximated as

$$
\kappa_{n}(y) \approx e^{-4(1-y)} .
$$

Next, $\rho_{n} \approx \operatorname{Var}\left[\delta_{i}[n]\right]$ in (14) is a constant that exclusively 
depends on the p.d.f. of the perturbation, i.e. $f_{\delta}\left(\delta_{i}\right)$. From all this, equation (10) can be approximately re-written as

$$
h_{n}(y) \stackrel{\Delta}{=} \mathrm{E}\left[\left(x_{n} y+x-y\right) \times \mathcal{H}\left(r_{n+1}-r_{n}\right) \mid \frac{1}{N} Y_{\text {best }}[n]=y\right] \text {, }
$$

where, to simplify notation, we have re-defined $r_{n}=R_{\text {best }}[n] / N$ and $r_{n+1}=R[n+1] / N$. From (6) and the fact that noise is stationary, it follows that

$$
\begin{gathered}
r_{n+1} \sim \mathcal{N}\left(\sqrt{\left(x_{n} y+x\right)^{2}+\frac{\sigma_{w}^{2}}{2 N^{2}}}, \frac{\sigma_{w}^{2}}{2 N^{2}}\right), \\
r_{n} \sim \mathcal{N}\left(\sqrt{y^{2}+\frac{\sigma_{w}^{2}}{2 N^{2}}, \frac{\sigma_{w}^{2}}{2 N^{2}}}\right),
\end{gathered}
$$

and, consequently, $z_{n+1} \stackrel{\Delta}{=} r_{n+1}-r_{n}$ equals

$$
z_{n+1} \sim \mathcal{N}\left(\sqrt{\left(x_{n} y+x\right)^{2}+\frac{\sigma_{w}^{2}}{2 N^{2}}}-\sqrt{y^{2}+\frac{\sigma_{w}^{2}}{2 N^{2}}}, \frac{\sigma_{w}^{2}}{N^{2}}\right)
$$

Finally, from (16) and (19), we conclude that the conditional expectation of the increase in beamforming gain equals:

$$
\begin{aligned}
& h_{n}(y)=\int_{-\infty}^{\infty} \int_{A(x)}^{\infty}\left(x-y\left(1-X_{n}\right)\right) \frac{1}{\sqrt{2 \pi \sigma_{\mathbb{R}}^{2}[n]}} e^{-\frac{x^{2}}{2 \sigma_{\mathbb{R}}^{2}[n]}} \times \\
& \times \frac{1}{\sqrt{2 \pi \sigma_{w}^{2} / N^{2}}} e^{-\frac{z^{2}}{2 \sigma_{w}^{2} / N^{2}}} d z d x \\
& =\int_{-\infty}^{\infty}\left(x-y\left(1-X_{n}\right)\right) \frac{1}{\sqrt{2 \pi \sigma_{\mathbb{R}}^{2}[n]}} e^{-\frac{x^{2}}{2 \sigma_{\mathbb{R}}^{2}[n]} \times} \\
& \times Q\left(\frac{\sqrt{y^{2}+\frac{\sigma_{w}^{2}}{2 N^{2}}}-\sqrt{\left(X_{n} y+x\right)^{2}+\frac{\sigma_{w}^{2}}{2 N^{2}}}}{\sigma_{w} / N}\right) d x,
\end{aligned}
$$

where the lower limit of the second integral equals:

$$
A(x)=\sqrt{y^{2}+\frac{\sigma_{w}^{2}}{2 N^{2}}}-\sqrt{\left(x_{n} y+x\right)^{2}+\frac{\sigma_{w}^{2}}{2 N^{2}}},
$$

and $Q(x)$ stands for the Q-function, namely,

$$
Q(x)=\frac{1}{\sqrt{2 \pi}} \int_{x}^{\infty} \exp \left(-u^{2} / 2\right) d u \text {. }
$$

Since a closed-form solution of (20) is difficult to obtain, in the sequel we will resort to numerical integration methods.

\section{B. Asymptotic behavior}

In [1] the authors proved that in a noiseless scenario the expected value of the normalized beamforming gain when $n \rightarrow \infty$ is $y=1$. Here, we show that in noisy scenarios such a limit is not achievable. Due to space limitations, only a sketch of the proof in [10] is included here. We start by noting that the increment of the normalized beamforming gain $h_{n}(y)$ is, by definition, a continuous function (actually, an integral of a continuous function). In [10], we prove on the one hand that $h_{n}(y)<0$ for $y=1$; and, on the other, that $h_{n}(y)>0$ for $y=0$. Hence, there exists a value of $y \in(0 \ldots 1)$ for which expected increase of the normalized beamforming gain turns out to be $h_{n}(y)=0$. At this point, the expected normalized beamforming gain saturates. Besides, when the recursive algorithm is initialized (i.e. before running the algorithm), the set of overall phase rotations $\Phi_{i}[n]$ are uniform i.i.d. random variables (over sensors). In these circumstances, one can easily show that the expected value of the beamforming gain is $1 / \sqrt{N}$. In other words, $1 / \sqrt{N}$ can be regarded as a lower bound which results when no control is exerted on the phases. For this reason, we conjecture that, in noisy scenarios, the maximum achievable value of the expected beamforming gain actually lies in the range $(1 / \sqrt{N} \ldots 1)$. As we will see, computer simulation results confirm this.

\section{Simultaneous Phase Synchronization AND DATA TRANSMISSION}

\section{A. Review of the one-bit of feedback algorithm}

In previous works [1], the main assumption is that the data are being transmitted after the sensors achieve full phase synchronization at the BS (see Fig. 2a). Depending on the number of sensors, the amount of energy and time required for carrier synchronization can be significant. As a consequence, we might lose the benefits of collaborative transmission in terms of energy efficiency.

In order to deal with this issue, here we propose an extension of the algorithm described in [1], where the sensors are allowed to transmit the common message before the full phase synchronization is achieved, as shown in Fig 2b. For the chosen PSK modulation scheme, the convergence rate of the proposed phase synchronization algorithm remains the same as in the scenario without simultaneous data transmission [1]. On the other hand, the phase synchronization algorithm has some impact on the bit error probability, $P_{e}$. This is due to the fact that the total phase of the received signal changes not only because of modulation, but also because of the random phase perturbations in synchronization algorithm.

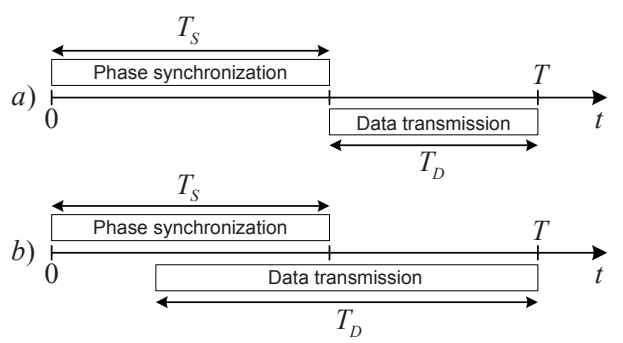

Fig. 2. Synchronization and data transmission periods: a)

Consecutive scheme, b) Simultaneous scheme.

\section{B. Communication protocol}

Upon BS request, the sensor nodes wake up for $T$ seconds during which a data packet will be transmitted. Typically, $T$ is predefined and turns out to be a small percentage of the time elapsed between consecutive requests. Within this period of time, the sensors need to (i) share the common message $m(t)$; (ii) synchronize their carriers; and (iii) actually transmit the message. For 
simplicity, we assume that (i) is carried out transparently to (ii) and (iii) and, hence, the packet consists of one synchronization block and one data transmission block only. Their respective durations are $T_{S}$ and $T_{D}$, with $T \leq T_{S}+T_{D}{ }^{4}$ and $T \geq \max \left\{T_{S}, T_{D}\right\}$. As shown in Fig. 2b, the data transmission starts before the carrier synchronization period is finished, this resulting in a more efficient usage of both time and energy. Nevertheless, the average BER of such transmission will depend on the iteration (and the corresponding instantaneous RSS level) where the data transmission has started. This, and the effect of other system parameters, will be analyzed in the sequel.

\section{BER analysis}

The coherent demodulation of BPSK signal requires a reference carrier signal to be generated at the receiver. This may not be feasible, either because additional complexity is required, or because the maximum delay for the given application cannot afford sufficient time for carrier synchronization.

One can overcome this problem by using the phases of two consecutive symbol intervals, and estimating their difference (DE-BPSK), as it has been done here. Namely, the binary " 1 " is transmitted by setting $v[n]=v[n-1]+\pi$, whereas the binary " 0 " is transmitted by setting $v[n]=v[n-1]$.

The $P_{e}$, for the case of DE-BPSK modulation and signal-to-noise ratio SNR, was shown in [11] [Sec 5.8.2] to be equal to $P_{e}=1 / 2 \times \mathrm{e}^{-\mathrm{SNR}}$, which for the case of collaborative beamforming can be expressed as:

$$
P_{e}=\frac{1}{2} e^{-\frac{Y^{2}}{\sigma_{\eta}^{2}}},
$$

with $\sigma_{\eta}^{2}$ including both additive Gaussian noise and the effect of phase perturbations. The p.d.f. of the beamforming gain $Y[n]$ in the $n$-th iteration is denoted by $p_{Y[n]}$, and it is evaluated numerically. Consequently, for the $n$-th iteration, the $P_{e}$ can be computed as:

$$
P_{e}[n]=\int_{0}^{\infty} \frac{1}{2} e^{-\frac{Y^{2}[n]}{\sigma_{\eta}^{2}[n]}} p_{Y[n]} d Y[n] .
$$

Finally, the BER for the total data transmission time $T_{D}$ can then be expressed as follows:

$$
\begin{aligned}
& \mathrm{BER}=\frac{1}{M_{D}} \sum_{n=M-M_{D}+1}^{M} P_{e}[n] \\
& =\frac{1}{2 M_{D}} \sum_{n=M-M_{D}+1}^{M} \int_{0}^{\infty} e^{-\frac{Y^{2}[n]}{\sigma_{\eta}^{2}[n]}} p_{Y[n]} d Y[n],
\end{aligned}
$$

with $M$ standing for the total number of iterations (timeslots) and $M_{D}$ denoting the number of timeslots during data transmission period.

\section{NUMERICAL RESULTS}

In the sequel, we present some analytical and computer simulation results illustrating the impact of channel noise

\footnotetext{
${ }^{4}$ Note that in [1] it is implicitly assumed that $T=T_{S}+T_{D}$. Here, however, the synchronization and data transmission periods overlap.
}

on the behavior of the algorithm and the performance of the proposed communication scheme. As for the analytical results, we employ equation (23) with $p_{Y[n]}$ evaluated numerically.

We assume that a cluster of wireless sensor nodes is deployed far away from the BS (e.g. to monitor environmental parameters in a remote area). Collected data are first shared among all the sensors in the cluster, and then transmitted to the remote BS by using collaborative beamforming. The total number of sensors equals $N=100$. Sensors are uniformly distributed over a disk of radius $R$ (normalized to the wavelength), and the phase perturbations for carrier synchronization are chosen independently among the sensors according to the uniform distribution $\delta_{i} \sim \mathcal{U}(-\pi / 50, \pi / 50)$.

In Fig. 3, we depict the evolution of the expected beamforming gain (normalized by the number of sensors). Several curves are shown for a collection of values of the noise variance. As a benchmark, the curve corresponding to a noiseless scenario $\left(\sigma_{w}^{2}=0\right)$ is included, as well. First of all, we observe a close match between computer simulations results (solid lines) and the prediction from our analysis in Section III (dashed lines). Next, and as discussed in Section III-B, we see that the algorithm does not achieve full beamforming gain in noisy scenarios (i.e. when $\left.\sigma_{w}^{2}>0\right)$. The explanation is that beyond some point, the fact that the RSS value upon which the decision on keeping or rejecting perturbations is affected by noise impedes the algorithm from fully aligning sensor phases (and, of course, the higher the noise, the lower the beamforming gain after convergence). Besides, this fact also results in a lower initial rate at which the algorithm converges (i.e. less steep curves around $n=0$ ).

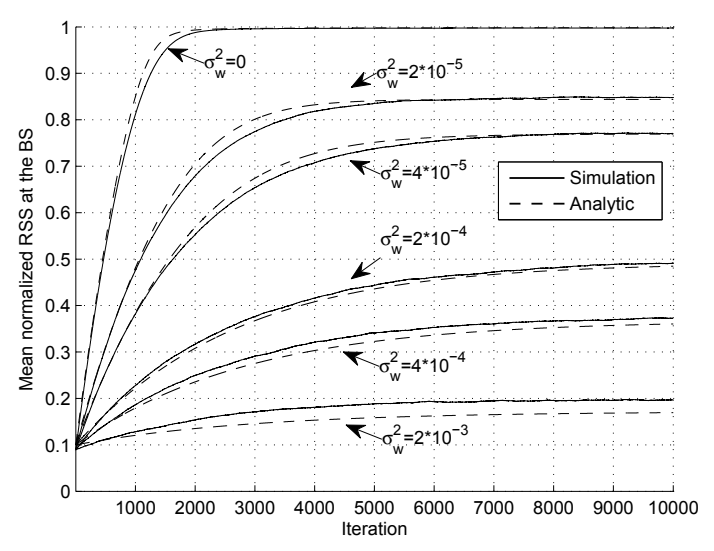

Fig. 3. Expected beamforming gain vs. number of iterations $\left(N=100, \delta_{i} \sim \mathcal{U}(-\pi / 50, \pi / 50)\right)$

Figure 4 presents the mean signal-to-noise ratio (SNR) as a function of algorithm's iteration for a collection of different noise powers $\sigma_{w}^{2}$. The plot reveals that the mean SNR increases slower when the noise power is higher. This behavior, as explained for Figure 3, is due to the impact of inaccurate RSS estimation at the BS on the decisions whether to keep or retain the phase perturbation.

In Figure 5, we illustrate the $P_{e}[n]$ as a function of algorithm's iteration, for different values of noise power at 
the BS. We provide both numerical simulation and semianalytical results (see eq. (23)), where the p.d.f. function of instantaneous beamforming gain levels $p_{Y[n]}$ is obtained from simulations. As we can see, semi-analytical results perfectly match the numerical simulations. Besides, we observe that for lower noise powers, the $P_{e}[n]$ decreases more rapidly with algorithm iterations. This is due to the fact that the low noise power implies faster phase synchronization, and consequently a much faster change of $P_{e}$ with iterations.

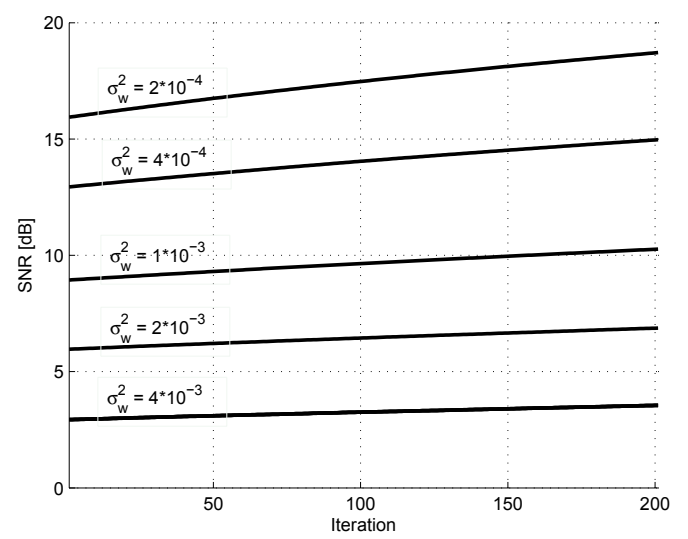

Fig. 4. The SNR as a function of algorithm's iteration $\left(N=100, \delta_{i} \sim \mathcal{U}(-\pi / 50, \pi / 50)\right)$

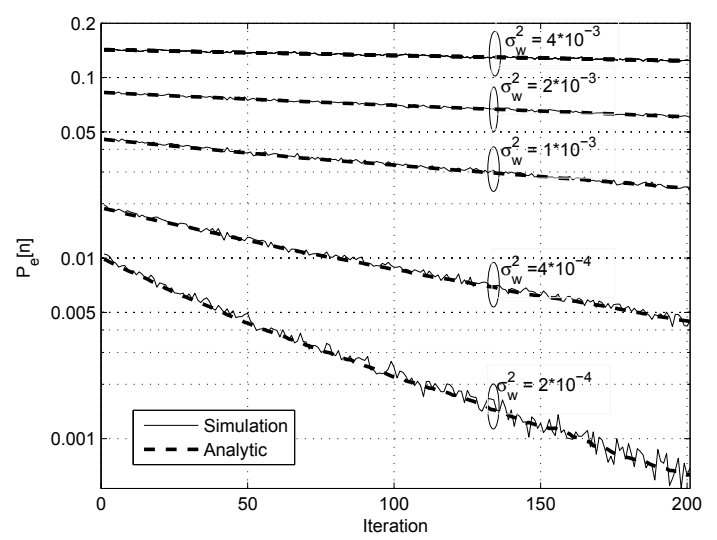

Fig. 5. $P_{e}[n]$ as a function of algorithm's iteration $\left(N=100, \delta_{i} \sim \mathcal{U}(-\pi / 50, \pi / 50)\right)$.

Finally, in Figure 6, we present the BER for the whole data transmission period as a function of the iteration where the sensors start transmitting data (starting iteration). When the data transmission starts later, the SNR levels during the data transmission are higher. As a consequence, the BERs for the whole data transmission period are lower. Again, the slopes of BER depend on algorithm convergence rate which is a function of the noise power.

\section{CONCLUSION}

In this paper, we have analyzed the impact of noise on the behavior of the collaborative beamforming scheme with one bit of feedback. We have seen that the noise prevents the algorithm from achieving a full beamforming gain and that it exhibits a slower convergence rate. In addition, we have proposed an extension of the algorithm for the case when carrier phase synchronization and data transmission are performed simultaneously. By using such a scheme, the synchronization overhead turns out to be shorter and consequently, the system is able to send more data in a more energy efficient manner.

The computer simulation results reveal that the BER as a function of algorithm's iteration decreases more rapidly when the noise power is lower, since in that case the algorithm's convergence rate is faster. Also, it can be concluded that depending on the given noise power and the target BER, there exists a suitable starting iteration for the data transmission period.

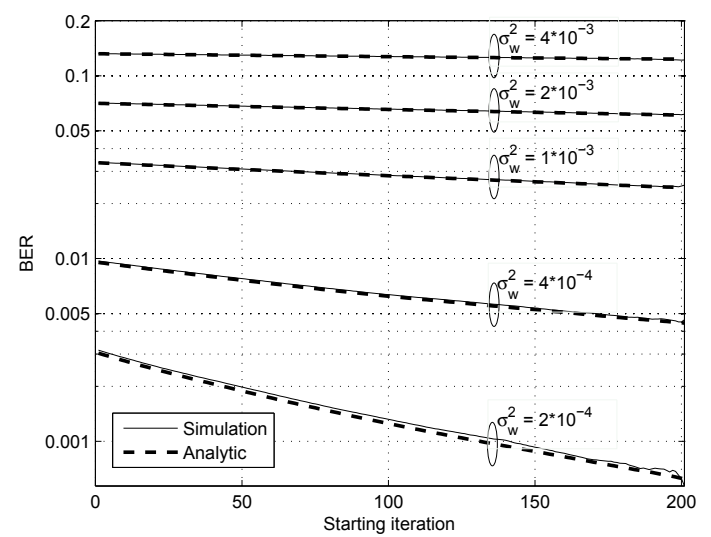

Fig. 6. The BER as a function of data transmission starting iteration $\left(N=100, \delta_{i} \sim \mathcal{U}(-\pi / 50, \pi / 50)\right)$.

\section{REFERENCES}

[1] R. Mudumbai, J. Hespanha, U. Madhow, and G. Barriac, "Distributed transmit beamforming using feedback control," IEEE Trans. on Inf. Theory, vol. 56, no. 1, pp. 411-426, January 2010.

[2] M. Seo, M. Rodwell, and U. Madhow, "A feedback-based distributed phased array technique and its application to 60-ghz wireless sensor network," IEEE MTT-S International Microwave Symposium Digest, pp. 683-686, June 2008.

[3] C. H.Wong, Z.W. Siew, R. Chin, A. Kiring, and K. Teo, "Adaptive phase synchronisation algorithm for collaborative beamforming in wireless sensor networks," in 7th Asia Modelling Symposium (AMS), 2013, July 2013, pp. 289-294.

[4] S. Song, J. Thompson, P.-J. Chung, and P. M. Grant, "Exploiting negative feedback information for one-bit feedback beamforming algorithm," IEEE Transactions on Wireless Communications, vol. 11, no. 2, pp. 516-525, February 2012.

[5] M.-O. Pun, D. Brown, and H. Vincent Poor, "Opportunistic collaborative beamforming with one-bit feedback," Proceeding of SPAWC 2008, pp. 246-250, July 2008.

[6] Y. Chen, H. Yang, X. Sun, and Z. Yang, "Opportunistic collaborative beamforming with phase-compensation and limited feedback," in IEEE Global Telecommunications Conference (GLOBECOM), Dec 2011, pp. 1-6.

[7] G. Mergen, Q. Zhao, and L. Tong, "Sensor networks with mobile access: Energy and capacity considerations," IEEE Transactions on Communications, vol. 54, no. 11, pp. 2033-2044, November 2006.

[8] L. Berbakov, C. Anton-Haro, and J. Matamoros, "Distributed beamforming with sidelobe control using one bit of feedback," in IEEE $73^{\text {rd }}$ Vehicular Technology Conference (VTC Spring 2011), May 2011, pp. 1-5.

[9] R. M. Henkelman, "Measurement of signal intensities in the presence of noise in MR images," Medical Physics, vol. 12, no. 2, pp. 232-233, March 1985.

[10] L. Berbakov, J. Matamoros, and C. Ant 'on-Haro, "Proof of the convergence of one-bit feedback algorithm for DBF in the presence of AWGN," CTTC Internal report, http://www.cttc.es/wp-content/ uploads/2013/03/111021-icassp-2012-one-bit-convergence-proofmerged-45467.pdf, sep. 2011..

[11] S. Benedetto and E. Biglieri, Principles of Digital Transmission with Wireless Applications. Kluwer Academic, New York, 1999. 